\title{
Sodium Selenite Accentuates the Therapeutic Effect of Adriamycin Prodrug (PADM) against Gastric Cancer
}

\author{
Shengquan Tan, ${ }^{1,2}$ Jiapeng Mo, ${ }^{2}$ Zixiong Zhang, ${ }^{2}$ Chuying Huang, ${ }^{2,3}$ Yi Zou, ${ }^{2}$ \\ and Jianhua Sun $\mathbb{D D}^{1,2}$ \\ ${ }^{1}$ Department of Gastrointestinal Surgery, The Central Hospital of Enshi Tujia and Miao Autonomous Prefecture, \\ No. 158 Wuyang Avenue, Enshi, Hubei 445000, China \\ ${ }^{2}$ Hubei Selenium and Human Health Institute, The Central Hospital of Enshi Tujia and Miao Autonomous Prefecture, \\ No. 158 Wuyang Avenue, Enshi, Hubei 445000, China \\ ${ }^{3}$ Department of Oncology, The Central Hospital of Enshi Tujia and Miao Autonomous Prefecture, No. 158 Wuyang Avenue, \\ Enshi, Hubei 445000, China \\ Correspondence should be addressed to Jianhua Sun; jhsun51@sina.com
}

Received 28 May 2019; Revised 9 September 2019; Accepted 11 September 2019; Published 13 October 2019

Guest Editor: Bin Duan

Copyright (C) 2019 Shengquan Tan et al. This is an open access article distributed under the Creative Commons Attribution License, which permits unrestricted use, distribution, and reproduction in any medium, provided the original work is properly cited.

Selenium has remained a controversial character in cancer research. While its antitumor effects have been widely demonstrated, further evidence is required to establish it as a robust treatment regime. Sodium selenite (SS), an inorganic selenium, reportedly affected the proliferation and redifferentiation of gastric cancer cells, but whether it could act as a complement to conventional chemotherapeutic drugs for combination therapy is uncertain. Herein, SGC-7901 and MGC-803 gastric cancer cells were treated with PADM (Ac-Phe-Lys-PABC-ADM), a prodrug of doxorubicin/adriamycin (ADM), and the combined antitumor effects of the two drugs were evaluated. Characterization after treatment revealed that although PADM exhibited antitumor effects individually by inhibiting the proliferation and migration of gastric cancer cells and inducing apoptosis, the addition of SS significantly amplified these effects. Furthermore, gastric cancer cell apoptosis triggered by the combined treatment of SS and PADM may involve the participation of mitochondrial apoptosis, as evidenced by the changes in mitochondrial morphology and occurrence of mitochondrial fission. Collectively, SS could be a strong complementary drug that accentuates the therapeutic potential of PADM in gastric cancer treatment and management, and its significance could contribute to unique and innovative anticancer strategies.

\section{Introduction}

With the rising incidence and high mortality of cancer, it has become the primary cause of death in China and emerged as a severe public health concern. Among various types of cancers, gastric cancer has the second highest rate of incidence and mortality in China. The total number of patients diagnosed with gastric cancer in China each year accounts for $42 \%$ of the worldwide number of cases, and the number of deaths exceeds two-thirds [1]. The strong heterogeneity of gastric cancer $[2,3]$ leads to a low rate of successful treatment, including surgical treatment, chemotherapy, radiotherapy, targeted therapy, and immunotherapy. Thus, optimizing the therapeutic scheme is an important step in the advancement of gastric cancer treatment.
Doxorubicin, or adriamycin (ADM), is one of the most important first-line drugs against cancer, with an effective rate of $40-50 \%$ when applied as a single-drug treatment regime. When $\mathrm{ADM}$ is combined with other chemotherapeutic drugs, it has an effective rate of up to $60-80 \%[4,5]$. Nevertheless, its clinical application is limited because its toxic effects increase with increasing dose [6,7]. To address this issue, an effective and low-toxicity chemotherapeutic prodrug has been developed in the form of the ADM precursor Ac-Phe-Lys-PABC-ADM (PADM) [8]. In healthy tissues and peripheral blood, PADM is inactive, and it is only activated in the presence of excess cathepsin B, which is overexpressed on cancer cell membranes. Upon activation, PADM is cleaved to release free ADM molecules, which then exert their intended therapeutic impact [8]. In this way, the 
toxicity of ADM is mitigated in healthy tissues, ensuring that the drug only targets cancer cells and is inactive otherwise.

In addition to chemotherapeutic drugs, other compounds with proven antitumor properties have also been considered in the development of optimal anticancer strategies. Among them, selenium is an important component of selenoproteins and a necessary trace element in the human body. Under normal physiological conditions, relatively high selenium content $(135 \mu \mathrm{g} / \mathrm{L})$ in the serum is positively related to the survival rate, whereas low selenium content $(87 \mu \mathrm{g} / \mathrm{L})$ in the serum increases the risk of cancer and death [9]. Selenium is also involved in spermatogenesis [10-12] and antagonizes cardiovascular diseases $[13,14]$ and tumor function $[15,16]$. Sodium selenite (SS), a common inorganic selenium, can induce superoxide anion production and lead to cancer cell apoptosis [17, 18]. SS has been highlighted as a promising alterative in cancer treatment, and its antitumor functions and therapeutic potentials warrant in-depth investigation.

In this study, the human gastric cancer cell lines SGC7901 and MGC-803 were subjected to combination treatment of SS and PADM. Cell proliferation, apoptosis, and mitochondrial morphology were evaluated to determine the therapeutic effect of SS and PADM. In turn, the effect and mechanism of the drug combination on the apoptosis of SGC-7901 and MGC-803 cells were explored.

\section{Materials and Methods}

2.1. Cell Culture and Protocol. The SGC-7901 cell line was purchased from Bena Culture Collection (100674), MGC803 cells were obtained from the Chinese Academy of Sciences, and GES- 1 cells were purchased from Procell. After the cells were thawed, they were centrifuged at $800 \mathrm{rpm}$ for 5 min to remove the supernatant and cultured in the RPMI1640 medium (HyClone, Logan, UT, USA) containing 10\% fetal bovine serum (FBS; Gibco; Thermo Fisher Scientific, MA, USA). Cells in the logarithmic growth were trypsinized with $0.25 \%$ trypsin (Bioswamp, Wuhan, China) in ethylenediaminetetraacetic acid and incubated in 6-well plates at $2 \times 10^{5}$ cells per well. The cells were divided into three treatment groups: (1) $30 \mu \mathrm{mol} / \mathrm{L}$ SS [19] for $24 \mathrm{~h}$, (2) $3.6 \mu \mathrm{g} / \mathrm{L}$ PADM [8] for $24 \mathrm{~h}$, and (3) $30 \mu \mathrm{mol} / \mathrm{L}$ SS for $24 \mathrm{~h}$ followed by $3.6 \mu \mathrm{g} / \mathrm{mL}$ PADM for $24 \mathrm{~h}$. Control SGC-7901 and MGC803 cells were not treated with either SS or PADM. The morphology of the cells was visualized using a light microscope (TS-100F; Nikon, Tokyo, Japan).

2.2. Colony Formation Assay. A suspension of SGC-7901 or MGC-803 cells was prepared using $0.25 \%$ trypsin, and the cells were cultured in the RPMI-1640 medium containing $10 \%$ FBS. Then, the cells were diluted at a gradient of 50, 100, and 200 cells in $10 \mathrm{~mL}$ of the preheated medium $\left(37^{\circ} \mathrm{C}\right)$ and shaken gently to ensure homogeneous distribution. The cells were cultured for three weeks at $37^{\circ} \mathrm{C}$ in an environment containing $5 \% \mathrm{CO}_{2}$ at saturated humidity. When visible clones were observed in the culture dish, the supernatant was discarded and the cells were washed twice with phosphate- buffered saline (PBS). The cells were then fixed with $5 \mathrm{~mL}$ of $4 \%$ paraformaldehyde for $15 \mathrm{~min}$, after which the fixative solution was removed and an appropriate amount of Giemsa solution was added to stain the cells for 10-30 min. The dye was rinsed with water, and the samples were dried. A light microscope (TS-100F; Nikon, Tokyo, Japan) was used to visualize the results.

2.3. Cell Scratch Assay. After SGC-7901 or MGC-803 cells were cultured in the serum-free medium in a 6 -well plate at $1 \times 10^{5}$ cells $/ \mathrm{mL}$ for $24 \mathrm{~h}$, SS and PADM were added to the cells for three days. Scratch lines were formed in the same direction using a $100 \mu \mathrm{L}$ pipette tip, and photos of the scratch were acquired under a microscope after $36 \mathrm{~h}$ of culture. The width of the scratch was determined using ImageJ.

2.4. Analysis of Cell Cycle and Apoptosis. Adherent SGC-7901 or MGC-803 cells were digested at $37^{\circ} \mathrm{C}$ in $0.25 \%$ trypsin (Bioswamp) and centrifuged at $1,000 \times g$ at $25^{\circ} \mathrm{C}$ for $5 \mathrm{~min}$. The supernatant was discarded, and the cells were washed three times with PBS. The cells $\left(1 \times 10^{5}\right.$ to $\left.5 \times 10^{5}\right)$ were collected and resuspended in $200 \mu \mathrm{L}$ of the binding buffer (cat. no. C1052-1; Beyotime Institute of Biotechnology, Haimen, China) for analysis using the Cell Cycle and Apoptosis Analysis Kit (Beyotime) according to the manufacturer's protocol. Annexin V-FITC/propidium iodide staining was carried out for apoptosis, whereas propidium iodide analysis was applied for cell cycle progression. Analyses were performed using the LSR II Flow Cytometry System equipped with FACSDiva software 4.1 (BD Biosciences, Franklin Lakes, NJ, USA). Data were analyzed with ModFit LT software package version 4.0 (Verity Software House, Inc., Topsham, ME, USA).

2.5. Western Blot. SGC-7901 or MGC-803 cells were centrifuged at $1,000 \times g$ for $5 \mathrm{~min}$ at $37^{\circ} \mathrm{C}$, washed three times with $1 \mathrm{~mL}$ of ice-cold PBS, and lysed using the Nuclear and Cytoplasmic Protein Extraction Kit (Beyotime) for $30 \mathrm{~min}$ at $4^{\circ} \mathrm{C}$. The cell lysate was centrifuged at $15,000 \times g$ at $4^{\circ} \mathrm{C}$ for $10 \mathrm{~min}$, and proteins were quantified using a bicinchoninic acid assay kit (Beyotime). A total of $15 \mu \mathrm{g}$ of proteins was separated by $10 \%$ sodium dodecyl sulfate-polyacrylamide gel electrophoresis, and the resolved proteins were transferred to a polyvinylidene fluoride membrane (EMD Millipore, Billerica, MA, USA). The membrane was blocked using 5\% skim milk powder at $4^{\circ} \mathrm{C}$ overnight and incubated overnight at $4^{\circ} \mathrm{C}$ with primary antibodies against the following proteins: GAPDH $(1: 2,500$; ab9485), cyclin-dependent kinase 4 (CDK4; 1 : 2,000; ab108357), Ki67 (1 : 5,000; ab92742), cyclin D1 (1 : 10,000; ab134175), cyclin E (1:1,000; ab33911), B-cell lymphoma 2 (Bcl-2; 1 : 1,000; ab32124), Bcl-2-associated X (Bax; 1:500; ab53154), cleaved caspase 3 (1:500; ab2302), cleaved caspase 9 (1:500; ab2324), p53 (1:1,000; ab131442), second mitochondria-derived activator of caspases (Smac; 1 : 1,$000 ;$ ab8114), and apoptotic protease activating factor 1 (APAF1; 1 : 1,1000; ab8114) (all from Abcam, Cambridge, UK). After three washes in PBS containing 0.05\% Tween 20 
for $5 \mathrm{~min}$ each, the membrane was incubated with horseradish peroxidase-conjugated goat anti-rabbit immunoglobulin G H\&L secondary antibody $(1: 2,000$; ab6721; Abcam) at $4^{\circ} \mathrm{C}$ overnight. The results were quantified by Molecular Imager ChemiDoc XRS+ System 2.0 (Bio-Rad Laboratories, Hercules, CA, USA).

2.6. Transmission Electron Microscopy. A cell suspension was prepared using $0.25 \%$ trypsin, and the cells were cultured in the RPMI-1640 medium containing 10\% FBS. Then, the cells were centrifuged at $500 \times g$ for $30 \mathrm{~s}$ at $37^{\circ} \mathrm{C}$. After the supernatant was discarded, the cells were fixed for $4 \mathrm{~h}$ in $2.5 \%$ glutaraldehyde in PBS and rinsed three times for $5 \mathrm{~min}$ each using $0.1 \mathrm{M}$ phosphoric acid. The fixed cells were dehydrated in ethanol at a concentration gradient (50\%, 70\%, and 90\%) for $20 \mathrm{~min}$ at each concentration and then in a mixture of $90 \%$ ethanol and $90 \%$ acetone $(1: 1)$ for $20 \mathrm{~min}$ at $4^{\circ} \mathrm{C}$ and $90 \%$ acetone for $20 \mathrm{~min}$ at $4^{\circ} \mathrm{C}$. The cells were sequentially embedded with a mixture of pure acetone and embedding liquid at $2: 1$ for $3 \mathrm{~h}$ at $25^{\circ} \mathrm{C}$, in a mixture of pure acetone and embedding liquid at $1: 2$ overnight at $25^{\circ} \mathrm{C}$, and in embedding liquid for $3 \mathrm{~h}$ at $37^{\circ} \mathrm{C}$. The specimen was solidified at $37^{\circ} \mathrm{C}$ overnight, $45^{\circ} \mathrm{C}$ for $12 \mathrm{~h}$, and then $60^{\circ} \mathrm{C}$ for $24 \mathrm{~h}$. The prepared specimen was cut at a thickness of $60 \mathrm{~nm}$ using an ultramicrotome (EM UC7; Leica, Solms, Germany), stained with 3\% uranyl acetate and lead citrate, and observed under a JEM-1200EX transmission electron microscope.

2.7. Statistical Analysis. Statistical analysis was conducted in SPSS 19.0 software (SPSS, Inc., Chicago, IL, USA) using the unpaired Student's $t$-tests. All experiments were performed in triplicate, and the data were expressed as mean \pm standard deviation (SD). $P<0.05$ was considered to be statistically significant.

\section{Results}

3.1. Inhibitory Effect of PADM on Gastric Cell Proliferation and Migration Was Amplified by SS. The effect of SS and PADM on normal cell survival and proliferation was first determined in a gastric epithelial cell line GES-1 using the CCK- 8 assay (Figure 1). GES-1 cells continued to proliferate for up to $48 \mathrm{~h}$ even after SS and/or PADM treatment, though at a slower rate than control cells. It is reasonable that the therapeutic agents have a certain degree of effect on normal cells, but they did not suppress normal cell proliferation. This indicates that SS and PADM are safe and nontoxic to normal cells, setting the foundation for our subsequent experiments using gastric cancer cell lines.

The proliferative potential of SGC-7901 and MGC-803 gastric cancer cells was then detected by the colony formation assay. Individual treatment of PADM decreased cell survival and proliferation (Figure 2(a)) and colony formation (Figure 2(b)), and this effect was accentuated with the combined treatment of SS and PADM. Furthermore, the migration of SGC-7901 and MGC-803 cells was reduced after treatment with SS or PADM, as demonstrated by the lower degree of gap closure in the

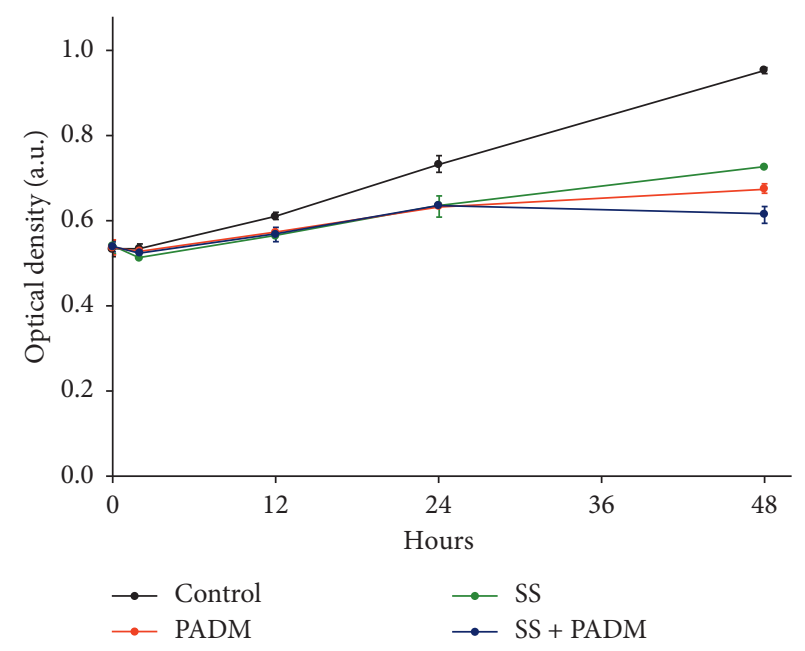

FIGURE 1: Effects of SS and PADM on normal gastric epithelial GES1 cells. The CCK- 8 assay was performed to determine the relative viability of GES- 1 cells after treatment with SS and/or PADM for 2, 12,24 , and $48 \mathrm{~h}$. All data are presented as mean \pm SD $(n=3)$.

scratched cell layer compared to that formed by nontreated cells (Figure 2(c)). Correspondingly, the combined antimigration effect of SS and PADM was greater than that of the individual components in both cell lines, suggesting that SS amplified the inhibitory potential of PADM in gastric cancer cell growth.

3.2. SS Promoted G1 Phase Blocking in Gastric Cancer Cells in conjunction with PADM. The cell cycle progression of SGC7901 and MGC-803 cells was evaluated by flow cytometry. Individual treatment of SS or PADM significantly reduced the percentage of cells in the G1 phase $(P<0.05)$, whereas that in the G2 phase was elevated compared with the control cells $(P<0.05)$. Effectively, combined treatment of SS and PADM exerted a greater impact on cell cycle progression than did the individual components $(P<0.05)$ (Figure 3(a)). This finding suggested that SS markedly enhanced the effect of PADM in inducing G1 phase blocking, in turn promoting cell cycle arrest at the $\mathrm{G} 2 / \mathrm{M}$ phase. Western blot revealed that the expression of the cell cycle-associated proteins CDK4, Ki67, cyclin E, and cyclin D1 was decreased after individual and combined treatment of SS and PADM (Figure 3(b)).

\subsection{SS Accentuated Apoptosis and Mitochondrial Fission in} PADM-Treated Gastric Cancer Cells. In accordance with the results of cell cycle progression, flow cytometry was performed to assess gastric cancer cell apoptosis (Figure 4(a)). Compared with nontreated control cells, the percentage of SGC-7901 and MGC-803 cell apoptosis was increased by individual administration of SS and PADM $(P>0.01)$, and their combined effect was amplified compared to that of individual treatment $(P<0.001)$. In turn, proteins associated with cell apoptosis (Bcl-2, Bax, cleaved caspase 3, and cleaved caspase 9) and mitochondrial apoptosis (p53, Smac, and APAF1) were examined by western blot. The 

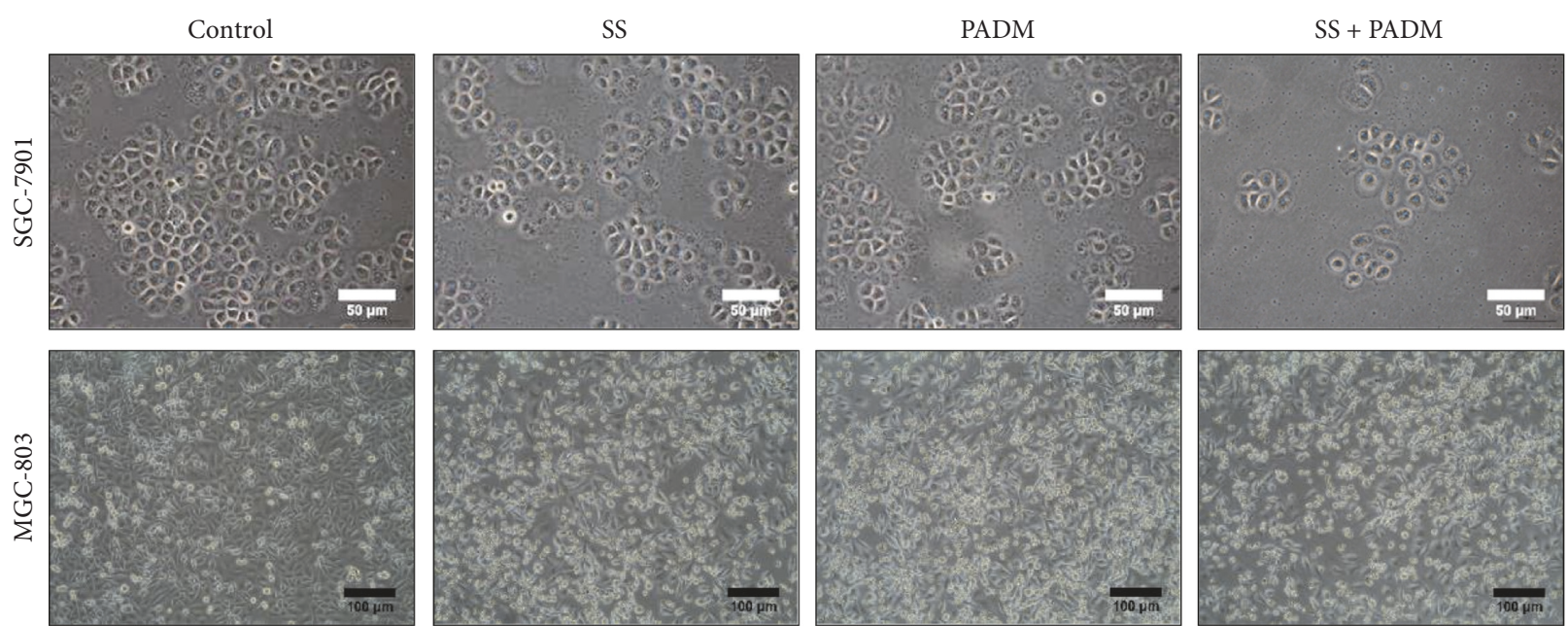

(a)
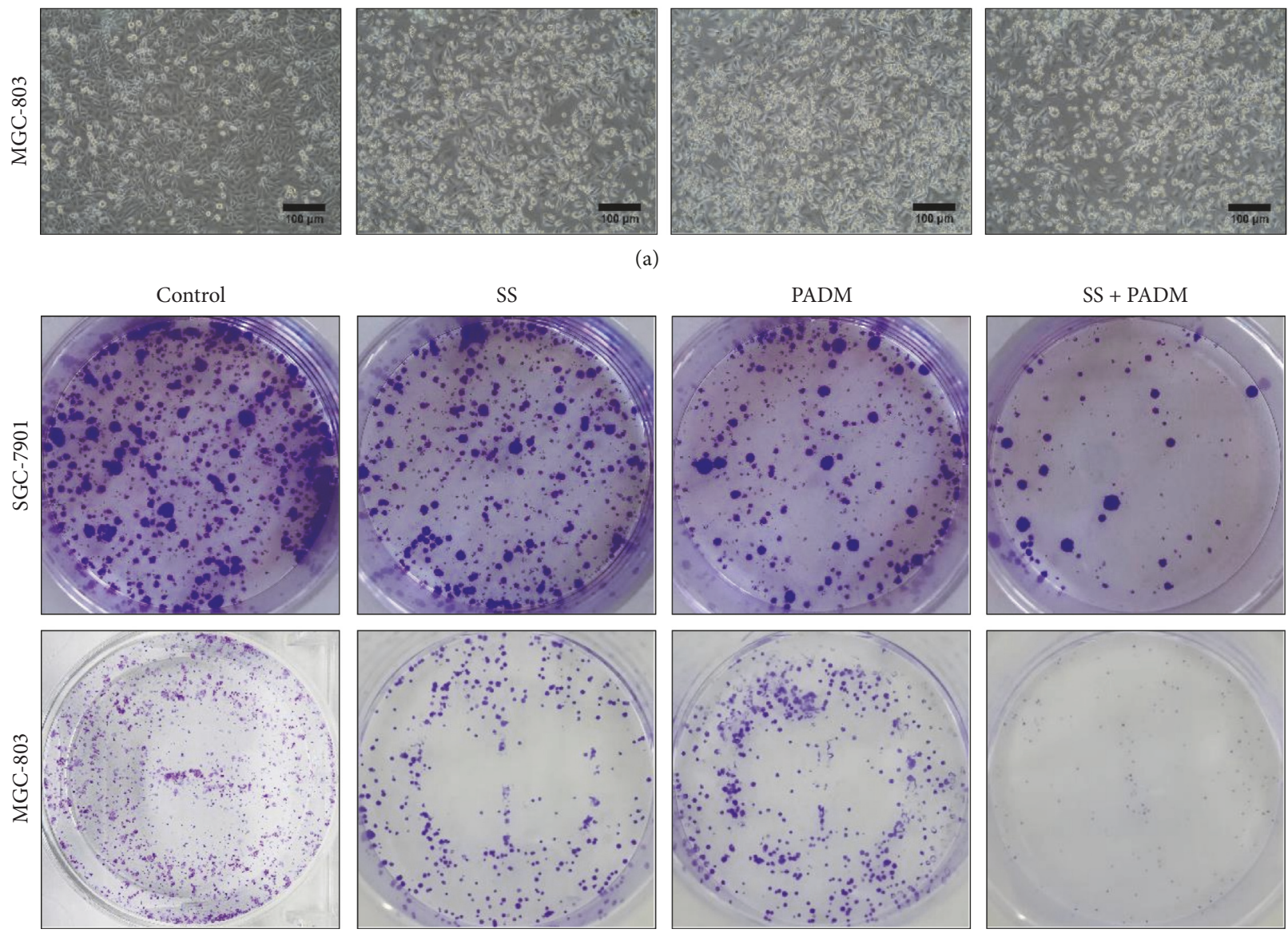

SS + PADM

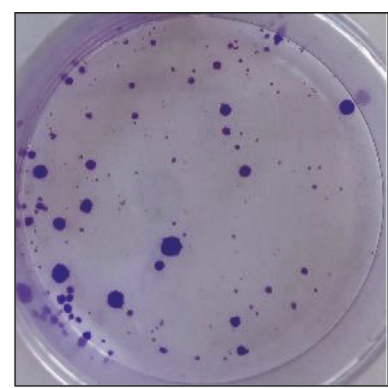

(b)

Figure 2: Continued. 


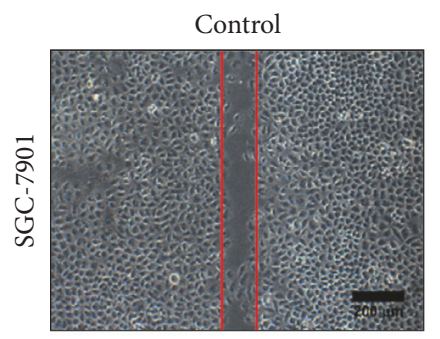

Width $=128 \mu \mathrm{m}$

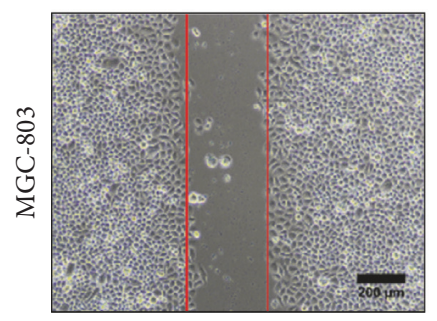

Width $=335 \mu \mathrm{m}$

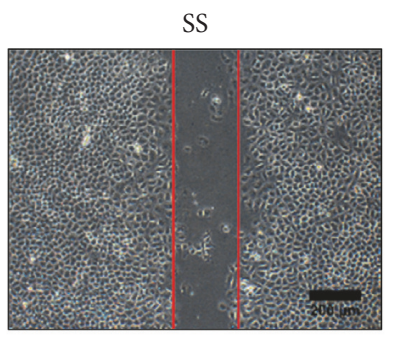

Width $=246 \mu \mathrm{m}$

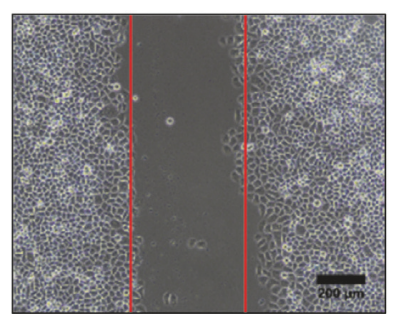

Width $=480 \mu \mathrm{m}$

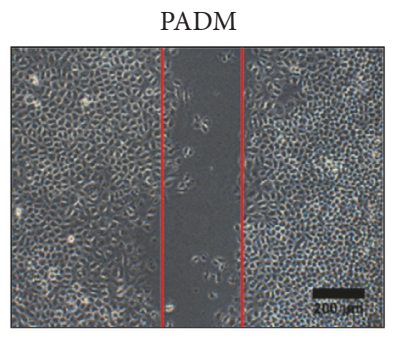

Width $=300 \mu \mathrm{m}$

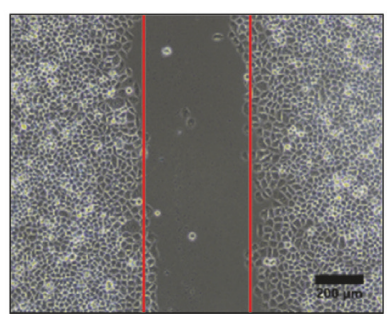

Width $=450 \mu \mathrm{m}$

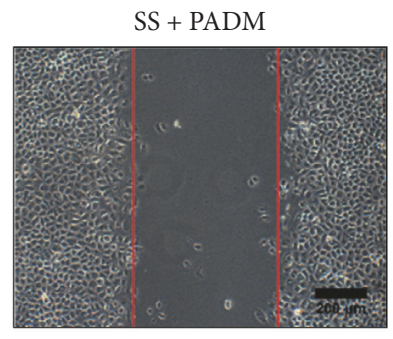

Width $=550 \mu \mathrm{m}$

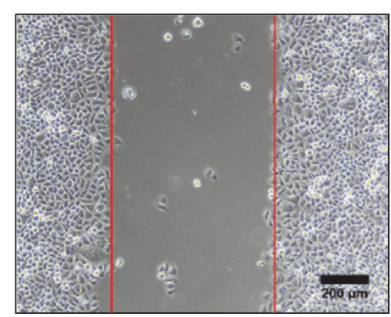

Width $=648 \mu \mathrm{m}$

(c)

FIGURE 2: Evaluation of the proliferation and migration of SGC-7901 and MGC-803 gastric cancer cells. (a) The proliferation of SGC-7901 and MGC-803 gastric cancer cells was observed using light microscopy. The number of SGC-7901 cells showed a decrease with SS and/or PADM treatment, and the number of dead MGC-803 cells (round, hollow cells) was increased with SS and/or PADM treatment. Scale bar $=50 \mu \mathrm{m}$ for SGC-7901 cells and $100 \mu \mathrm{m}$ for MGC-803 cells. (b) The proliferation of SGC-7901 and MGC-803 cells was detected by the colony formation assay (magnification 40x). Both SGC-7901 and MGC-803 cells showed a clear decrease in colony formation with SS or PADM treatment, and the combination of SS and PADM accentuated the effects of the individual components. (c) The migration of SGC7901 and MGC-803 cells was determined by the scratch assay. Gap closure efficiency was reduced by SS or PADM in both cell lines, and the combination of SS and PADM accentuated the effects of the individual components. Scale bar $=200 \mu \mathrm{m}$ for both cell lines.
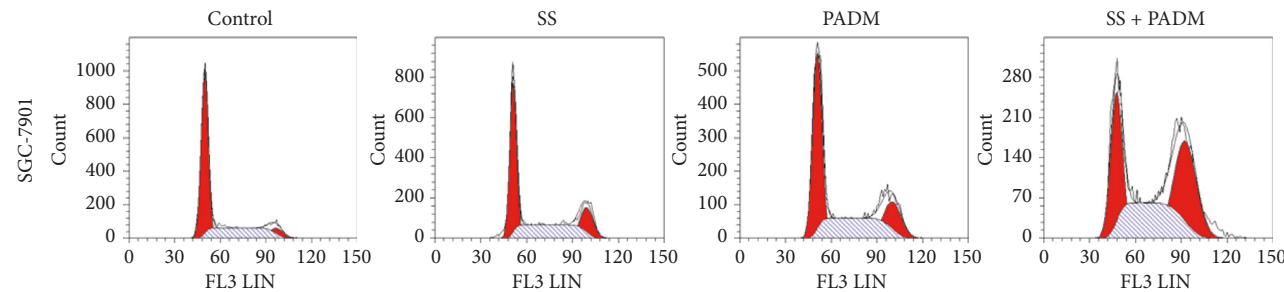

FL3 LIN

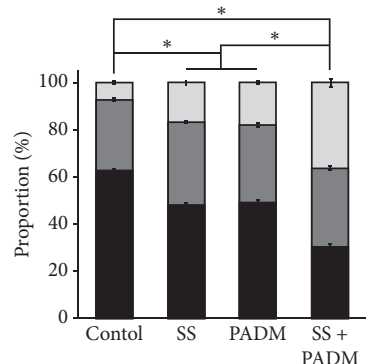

$\square \mathrm{G} 2$

$\square S$

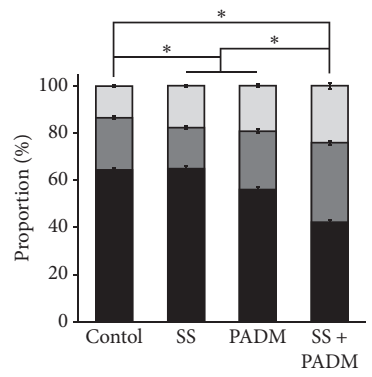

$\square$ G2

$\square \mathrm{S}$

(a)

Figure 3: Continued. 

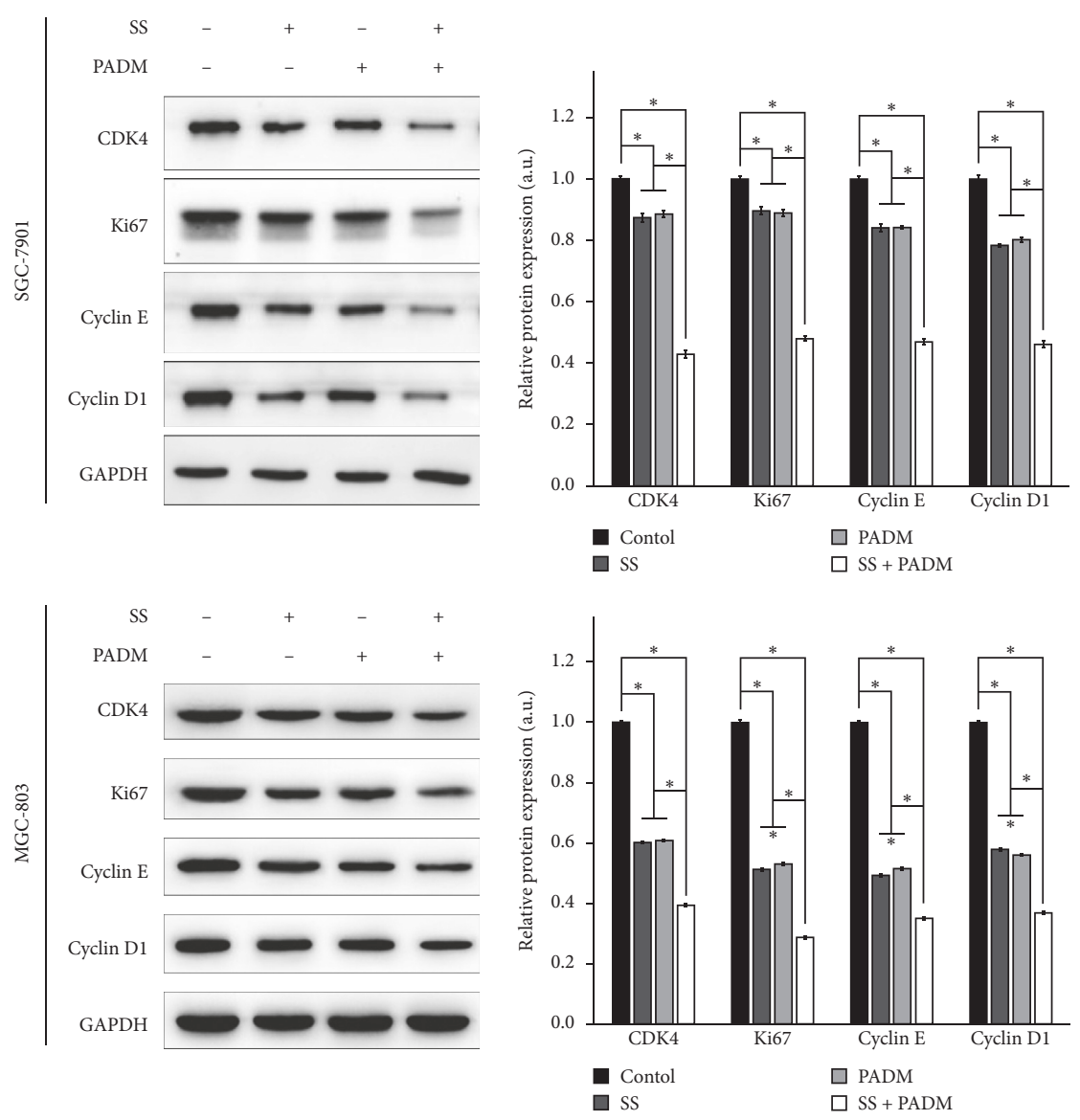

(b)

FIGURE 3: G1 phase blocking and cell cycle progression in SGC-7901 and MGC-803 cells. (a) Cell cycle progression in SGC-7901 and MGC803 cells was detected by flow cytometry. G1 phase blocking was observed in both cell lines when treated with SS or PADM, and the combined effect of SS and PADM was greater than that of the individual components. (b) Proteins associated with cell cycle (CDK4, Ki67, cyclin E, and cyclin D1) were examined by western blot. Decreased expression of these proteins was observed in both cell lines when treated with SS or PADM, and the combined effect of SS and PADM was greater than that of the individual components. All data are presented as mean $\pm \mathrm{SD}(n=3) .{ }^{*} P<0.05$.
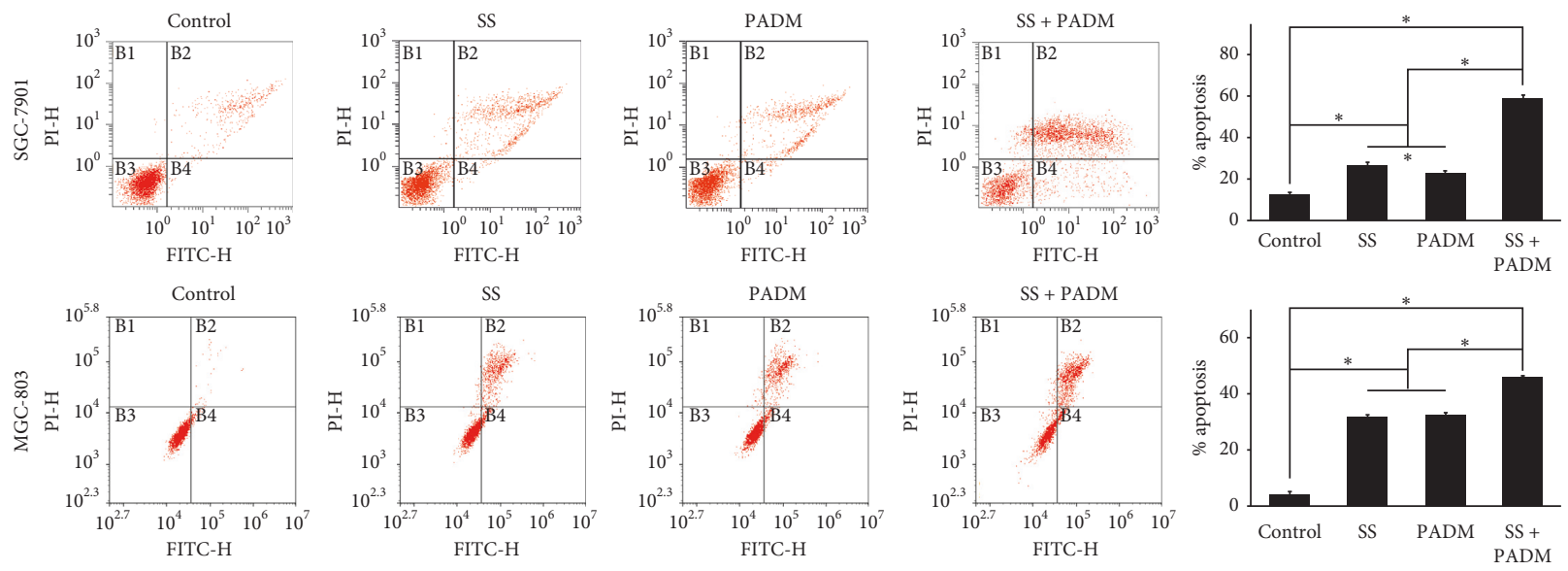

(a)

Figure 4: Continued. 

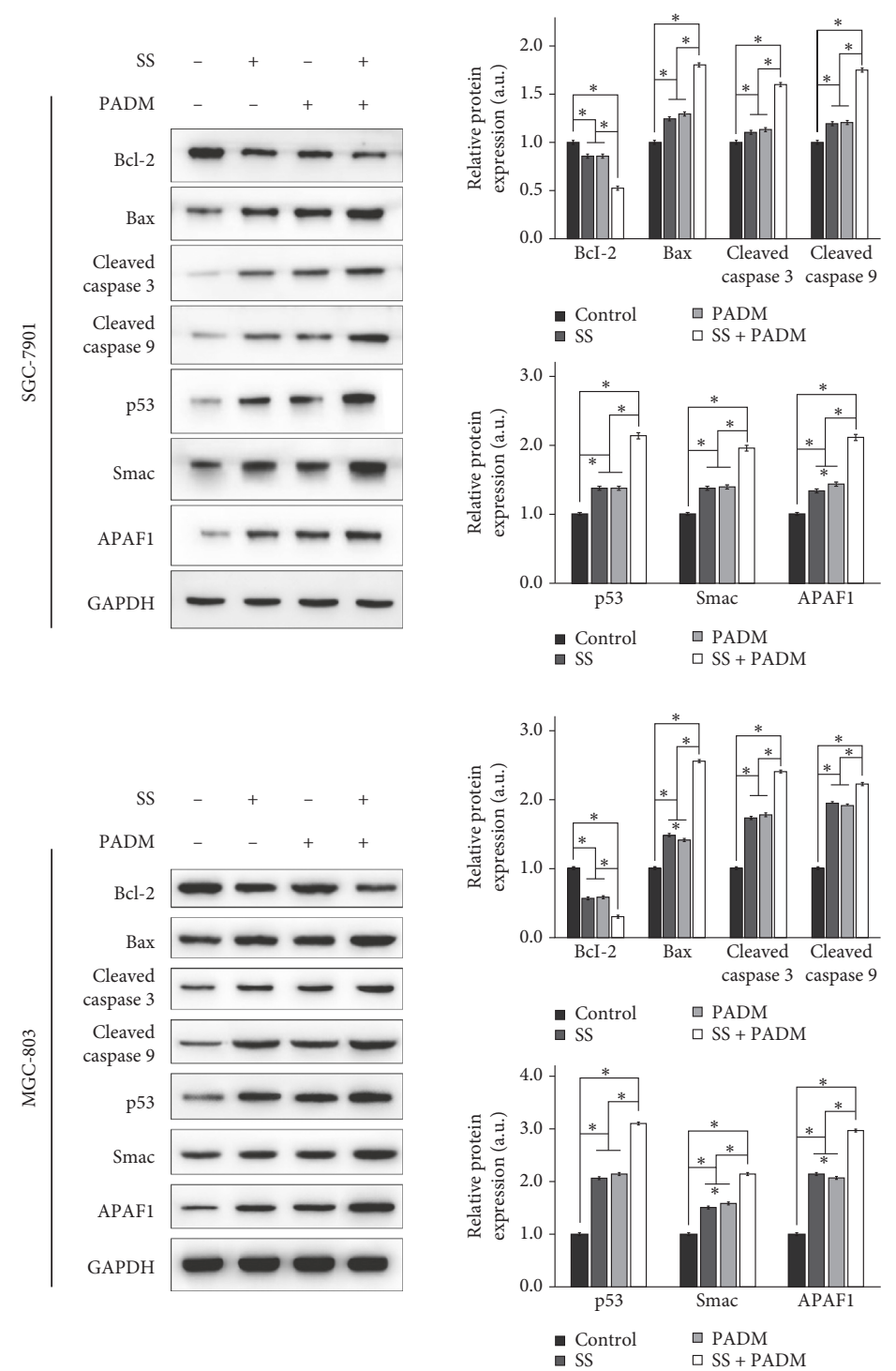

(b)

Figure 4: Apoptosis of SGC-7901 and MGC-803 cells. (a) The percentage of SGC-7901 and MGC-803 cell apoptosis was detected by flow cytometry. The percentage of apoptotic cells was increased in both cell lines when treated with SS or PADM, and the combined effect of SS and PADM was greater than that of the individual components. (b) Proteins associated with apoptosis (Bcl-2, Bax, cleaved caspase 3, and cleaved caspase 9) and mitochondrial apoptosis (p53, Smac, and APAF1) were examined by western blot. Downregulation of Bcl-2 and upregulation of Bax, cleaved caspase 3, cleaved caspase 9, p53, Smac, and APAF1 were observed in both cell lines after treatment with SS or $\mathrm{PADM}$, and the combined effect of SS and PADM was greater than that of the individual components. All data are presented as mean \pm SD $(n=3) .{ }^{*} P<0.05$.

expression of the antiapoptosis protein $\mathrm{Bcl}-2$ was downregulated, whereas the expression levels of the proapoptotic Bax, cleaved caspase 3, and cleaved caspase 9 were upregulated by combined SS and PADM treatment. Similarly, p53, Smac, and APAF1 were significantly upregulated by combination treatment compared with individual treatment (Figure 4(b)).

To examine the role of mitochondrial function more closely, the mitochondria in treated SGC-7901 cells were observed by transmission electron microscopy (Figure 5). The subcellular mitochondrial morphology was normal in the control cells, wherein mitochondria were present in an oval, elongated shape. However, mitochondrial fission appeared to occur when the cells were treated with PADM or SS. This was demonstrated by the individual round mitochondria that are close to each other, which could be indicative of the fission process and implicate mitochondrial apoptosis. When SS was administered in conjunction with PADM, mitochondrial fission became more obvious than in the cases of individual treatment. Collectively, these data suggested that the addition of SS accentuated the effect of PADM in causing apoptosis in SGC-7901 cells, and the mitochondrial apoptosis pathway may be involved in this process. 
Control

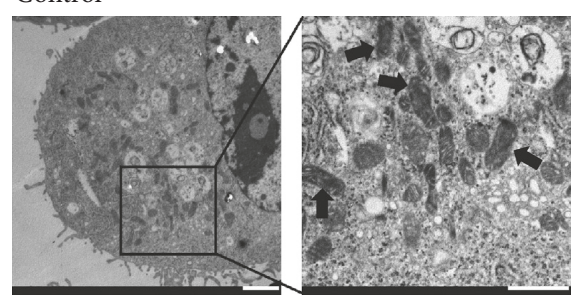

(a)

PADM

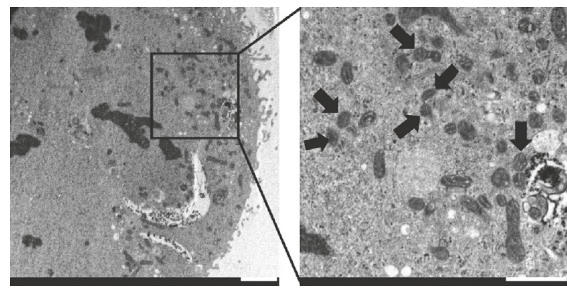

(c)

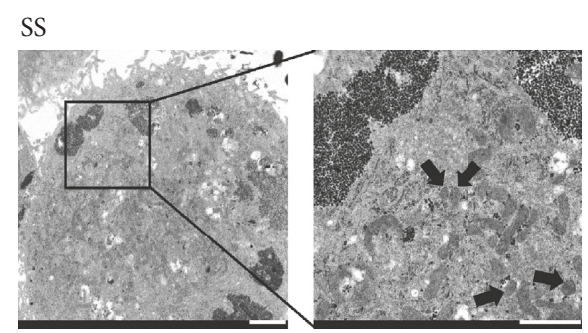

(b)

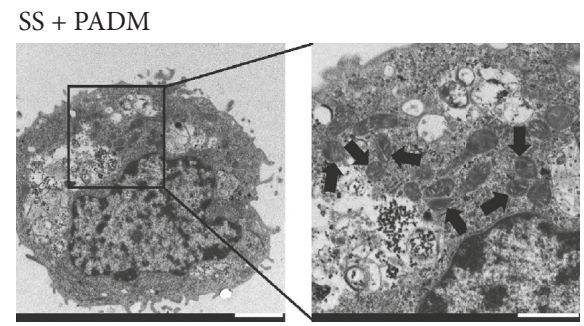

(d)

FIgURE 5: Morphology of subcellular mitochondria in SGC-7901 cells. The morphology and structure of mitochondria were observed by transmission electron microscopy. Dark, oval, or round features in the images represent mitochondria. In each group, a magnified image of the rectangular area is shown. Black arrows in the magnified images represent subcellular mitochondria. Scale bar $=2 \mu \mathrm{m}$ and $1 \mu \mathrm{m}$ (for magnification).

\section{Discussion}

PADM has recently emerged as an alternative to ADM, offering a low-toxicity strategy in the treatment of gastric cancer. In the PADM molecule, the specific substrate AcPhe-Lys is linked to ADM via PABC (para-aminobenzyloxycarbonyl), which acts as a spacer. Upon exposure to cathepsin $B$, which is present on cancer cell membranes, the Lys-PABC bond is cleaved, and subsequent self-hydrolysis of PABC allows free ADM to be released [8]. Cathepsin $B$ is an important class of enzymes related to tumor progression and is a candidate target for molecular therapy. The related characteristics of cathepsin B have been applied in the development of new anticancer drugs based on molecular targeting [20]. The use of PADM takes advantage of the biological characteristics of local cathepsin B release in the process of gastric cancer invasion and metastasis [21], demonstrating that the toxicity of PADM was significantly reduced under exposure to low levels of cathepsin B. In turn, safe doses of PADM prevented the peritoneal metastasis of gastric cancer in nude mice, reduced toxicity to the heart, liver, and kidney, and suppressed the overall toxic and side effects [8].

Selenium is a strong antioxidant with proven anticancer properties, but several selenoproteins, such as thioredoxin reductase 1 and selenoprotein 15, have been shown to exhibit dual tumor-promoting and tumor-suppressive functions [22]. In cancer research, selenium has provoked controversy as a treatment scheme, and opinions have been split as to whether the use of selenium is beneficial in cancer prevention. Some studies have reported on the prominent link between selenium deficiency and cancer incidence $[23,24]$, but null association has been reported in others $[25,26]$. Regional variations may also need to be taken into consideration when addressing this issue. Taken together, there is a lack of evidence drawing a robust link between selenium and cancer. With the emergence of combination therapy as a highlight in tumor treatment and management, various compounds have been suggested as complements to conventional chemotherapeutic drugs, either to maximize the effects or to minimize the toxicity of these drugs. Among these compounds, SS has gained attention as a promising candidate, and its safety and efficacy have been evaluated in clinical pharmacokinetic studies [27]. At a dose lower than $2 \mu \mathrm{M}$, SS promoted cell survival, while a hypertrophic dose of selenium (higher than $5 \mu \mathrm{M}$ ) induced apoptosis in a variety of tumor cells [28]. Selenite can also selectively eliminate tumor cells and have few side effects on normal cells. The intravenous injection of selenite containing $1.5-4.0 \mu \mathrm{C} / \mathrm{kg}$ ${ }^{75}$ Se into patients harboring various types of tumors showed that selenite was selectively enriched in patients with intracranial and thoracic and abdominal tumors [29]. In addition, studies have shown that SS is effective in killing cancer cells [30] and is closely related to the production of reactive oxygen species [31].

This study focuses on the important role of SS in accentuating the therapeutic potential of PADM, aiming to achieve the goal of minimal toxicity and maximized efficacy against tumor cells. While PADM alone was able to promote gastric cancer cell apoptosis, SS acts as a complementary agent to further emphasize the antitumor effects. The combined treatment strategy has advantages that contribute to the development of anticancer therapeutics. In particular, the synergistic effects of SS and PADM are stronger than those of the individual components, and in the meantime, toxicity induced by conventional drugs such as ADM could be mitigated with the use of prodrugs, PADM in this case. The findings of this study also elucidate that the apoptotic 
mechanisms were due to an increase in mitochondrial fragmentation through the process of fission, which is a required step in apoptosis [32]. Moreover, the activation of caspases 3 and 9 has been associated with mitochondrial remodeling and apoptosis [33]. The upregulation of cleaved caspases 3 and 9 by SS and PADM not only serves as a sign of cell apoptosis but also supports the hypothesis that the mitochondrial apoptosis pathway is a vital component of the antitumor effects of the proposed combination therapy.

\section{Conclusions}

The findings of this study presented a novel strategy of combination therapy (SS and PADM), which inhibited the proliferation and promoted the apoptosis of gastric cancer cells. Importantly, the prominent role of SS in accentuating the therapeutic effects of PADM was demonstrated from various perspectives and implicates the involvement of mitochondrial apoptotic pathways through the fission process. The specific mechanisms and signaling pathways involved in the combination treatment of SS and PADM in vitro require additional exploration.

\section{Data Availability}

The data used to support the findings of this study are available from the corresponding author upon request.

\section{Conflicts of Interest}

The authors declare that they have no conflicts of interest.

\section{Authors' Contributions}

Shengquan Tan and Jiapeng Mo contributed equally to this work.

\section{Acknowledgments}

This study was funded by the Hubei Natural Science Foundation (no. 2016CFB668) and the Scientific Research Project of The Central Hospital of Enshi Tujia and Miao Autonomous Prefecture "Selenium and Human Health" (no. 201500001).

\section{References}

[1] W. Chen, R. Zheng, P. D. Baade et al., "Cancer statistics in China, 2015," CA: A Cancer Journal for Clinicians, vol. 66, no. 2, pp. 115-132, 2016.

[2] P. Hudler, "Challenges of deciphering gastric cancer heterogeneity," World Journal of Gastroenterology, vol. 21, no. 37, pp. 10510-10527, 2015.

[3] P. Stahl, C. Seeschaaf, P. Lebok et al., "Heterogeneity of amplification of HER2, EGFR, CCND1 and MYC in gastric cancer," BMC Gastroenterology, vol. 15, no. 1, p. 7, 2015.

[4] M. Levin, S. Durgam, and A. Novetsky, "Cyclophosphamide, doxorubicin, and gemcitabine combination chemotherapy for treatment of metastatic and locally advanced breast cancer," Cancer Investigation, vol. 20, no. 7-8, pp. 872-875, 2002.
[5] H. T. Mouridsen, "Systemic therapy of advanced breast cancer," Drugs, vol. 44, no. 4, pp. 17-28, 1992.

[6] D. B. Sawyer, "Anthracyclines and heart failure," New England Journal of Medicine, vol. 368, no. 12, pp. 1154-1156, 2013.

[7] G. Takemura and H. Fujiwara, "Doxorubicin-induced cardiomyopathy from the cardiotoxic mechanisms to management," Progress in Cardiovascular Diseases, vol. 49, no. 5, pp. 330-352, 2007.

[8] L.-H. Shao, S.-P. Liu, J.-X. Hou et al., "Cathepsin B cleavable novel prodrug Ac-Phe-Lys-PABC-ADM enhances efficacy at reduced toxicity in treating gastric cancer peritoneal carcinomatosis: an experimental study," Cancer, vol. 118, no. 11, pp. 2986-2996, 2012.

[9] N. T. Akbaraly, J. Arnaud, I. Hininger-Favier, V. Gourlet, A. M. Roussel, and C. Berr, "Selenium and mortality in the elderly: results from the EVA study," Clinical Chemistry, vol. 51, no. 11, pp. 2117-2123, 2005.

[10] N. S. Alabi, M. A. Beilstein, and P. D. Whanger, "Chemical forms of selenium present in rat and ram spermatozoa: in vivo and in vitro studies," Biological Trace Element Research, vol. 76, no. 2, pp. 161-173, 2000.

[11] E. Ernst and J. G. Lauritsen, "Effect of organic and inorganic mercury on human sperm motility," Pharmacology \& Toxicology, vol. 68, no. 6, pp. 440-444, 1991.

[12] J. Marin-Guzman, D. C. Mahan, and R. Whitmoyer, "Effect of dietary selenium and vitamin $\mathrm{E}$ on the ultrastructure and ATP concentration of boar spermatozoa, and the efficacy of added sodium selenite in extended semen on sperm motility," Journal of Animal Science, vol. 78, no. 6, pp. 1544-1550, 2000.

[13] B. L. Dhanya, R. P. Swathy, and M. Indira, "Selenium downregulates oxidative stress-induced activation of leukotriene pathway in experimental rats with diabetic cardiac hypertrophy," Biological Trace Element Research, vol. 161, no. 1, pp. 107-115, 2014.

[14] K. Kalishwaralal, S. Jeyabharathi, K. Sundar, and A. Muthukumaran, "Comparative analysis of cardiovascular effects of selenium nanoparticles and sodium selenite in zebrafish embryos," Artificial Cells, Nanomedicine, and Biotechnology, vol. 44, no. 3, pp. 990-996, 2016.

[15] P. B. Caffrey and G. D. Frenkel, "Prevention of carboplatininduced resistance in human ovarian tumor xenografts by selenite," Anticancer Research, vol. 33, no. 10, pp. 4249-4254, 2013.

[16] Y. Wang, H. Hao, H. Liu et al., "Selenite-releasing bone mineral nanoparticles retard bone tumor growth and improve healthy tissue functions in vivo," Advanced Healthcare Materials, vol. 4, no. 12, pp. 1813-1818, 2015.

[17] A. P. Fernandes, M. Wallenberg, V. Gandin et al., "Methylselenol formed by spontaneous methylation of selenide is a superior selenium substrate to the thioredoxin and glutaredoxin systems," PLoS One, vol. 7, no. 11, Article ID e50727, 2012.

[18] L. Fu, X. Yan, X. Ruan, J. Lin, and Y. Wang, "Differential protein expression of Caco-2 cells treated with selenium nanoparticles compared with sodium selenite and selenomethionine," Nanoscale Research Letters, vol. 9, no. 1, p. 589, 2014.

[19] J. Gong and L. Li, "Sodium selenite inhibits proliferation of gastric cancer cells by inducing SBP1 expression," The Tohoku Journal of Experimental Medicine, vol. 239, no. 4, pp. 279-285, 2016.

[20] M. A. Nouh, M. M. Mohamed, M. El-Shinawi et al., "Cathepsin B: a potential prognostic marker for inflammatory 
breast cancer," Journal of Translational Medicine, vol. 9, no. 1, p. 1, 2011.

[21] G. M. Dubowchik, R. A. Firestone, L. Padilla et al., "Cathepsin B-labile dipeptide linkers for lysosomal release of doxorubicin from internalizing immunoconjugates: model studies of enzymatic drug release and antigen-specific in vitro anticancer activity," Bioconjugate Chemistry, vol. 13, no. 4, pp. 855-869, 2002.

[22] D. L. Hatfield, M.-H. Yoo, B. A. Carlson, and V. N. Gladyshev, "Selenoproteins that function in cancer prevention and promotion," Biochimica et Biophysica Acta (BBA)-General Subjects, vol. 1790, no. 11, pp. 1541-1545, 2009.

[23] M. B. Irmak, G. Ince, M. Ozturk, and R. Cetin-Atalay, "Acquired tolerance of hepatocellular carcinoma cells to selenium deficiency: a selective survival mechanism?," Cancer Research, vol. 63, no. 20, pp. 6707-6715, 2003.

[24] D. J. Hughes, V. Fedirko, M. Jenab et al., "Selenium status is associated with colorectal cancer risk in the European prospective investigation of cancer and nutrition cohort," International Journal of Cancer, vol. 136, no. 5, pp. 1149-1161, 2015.

[25] S. Männistö, G. Alfthan, M. Virtanen, V. Kataja, M. Uusitupa, and P. Pietinen, "Toenail selenium and breast cancer-a casecontrol study in Finland," European Journal of Clinical Nutrition, vol. 54, no. 2, pp. 98-103, 2000.

[26] D. L. McCormick, K. V. N. Rao, W. D. Johnson, M. C. Bosland, R. A. Lubet, and V. E. Steele, "Null activity of selenium and vitamin $\mathrm{E}$ as cancer chemopreventive agents in the rat prostate," Cancer Prevention Research, vol. 3, no. 3, pp. 381-392, 2010.

[27] O. Brodin, S. Eksborg, M. Wallenberg et al., "Pharmacokinetics and toxicity of sodium selenite in the treatment of patients with carcinoma in a phase I clinical trial: the SECAR study," Nutrients, vol. 7, no. 6, pp. 4978-4994, 2015.

[28] L. Guan, B. Han, J. Li et al., "Exposure of human leukemia $\mathrm{NB}_{4}$ cells to increasing concentrations of selenite switches the signaling from pro-survival to pro-apoptosis," Annals of Hematology, vol. 88, no. 8, pp. 733-742, 2009.

[29] R. R. Cavalieri, K. G. Scott, and E. Sairenji, "Selenite (75Se) as a tumor-localizing agent in man," Journal of Nuclear Medicine: Official Publication, Society of Nuclear Medicine, vol. 7, no. 3, pp. 197-208, 1966.

[30] L. Björkhem-Bergman, K. Jönsson, L. C. Eriksson et al., "Drug-resistant human lung cancer cells are more sensitive to selenium cytotoxicity: effects on thioredoxin reductase and glutathione reductase," Biochemical Pharmacology, vol. 63, no. 10, pp. 1875-1884, 2002.

[31] H.-M. Shen, C.-F. Yang, and C.-N. Ong, "Sodium seleniteinduced oxidative stress and apoptosis in human hepatoma $\mathrm{HepG}_{2}$ cells," International Journal of Cancer, vol. 81, no. 5, pp. 820-828, 1999.

[32] R. J. Youle and M. Karbowski, "Mitochondrial fission in apoptosis," Nature Reviews Molecular Cell Biology, vol. 6, no. 8, pp. 657-663, 2005.

[33] M. Brentnall, L. Rodriguez-Menocal, R. De Guevara, E. Cepero, and L. H. Boise, "Caspase-9, caspase-3 and caspase7 have distinct roles during intrinsic apoptosis," BMC Cell Biology, vol. 14, no. 1, p. 32, 2013. 


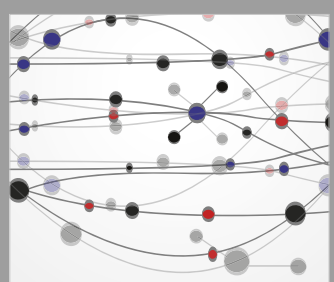

The Scientific World Journal
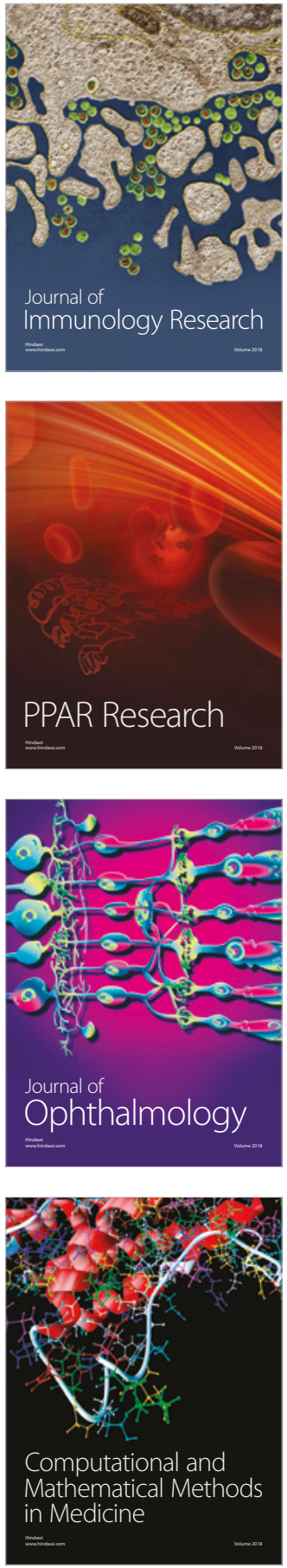

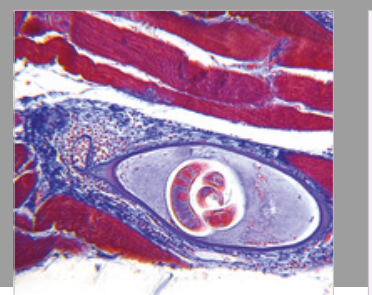

Gastroenterology Research and Practice

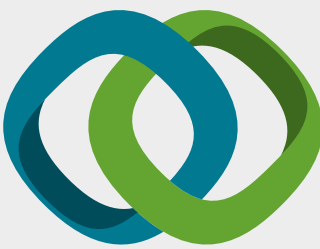

\section{Hindawi}

Submit your manuscripts at

www.hindawi.com
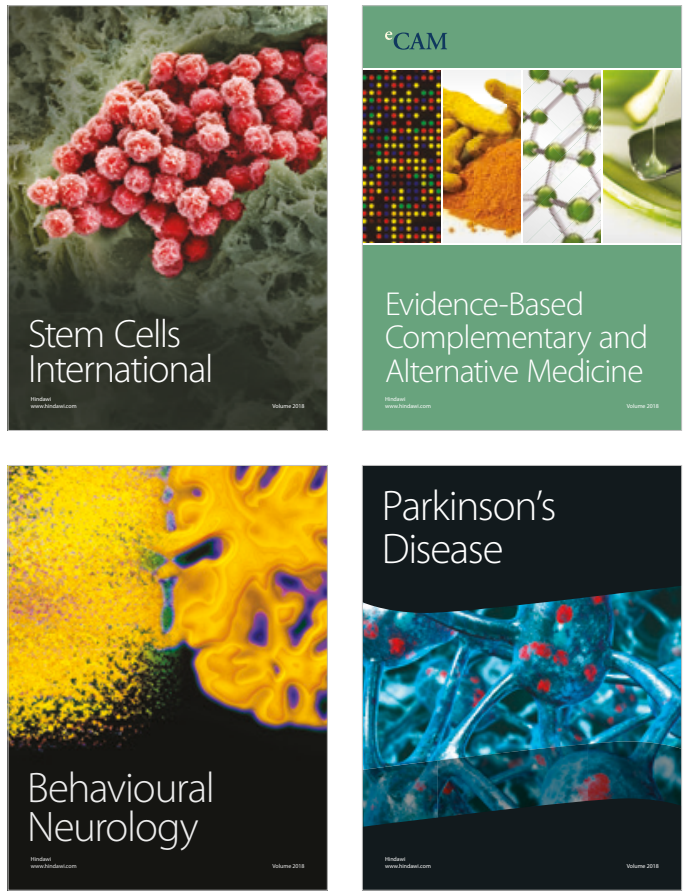

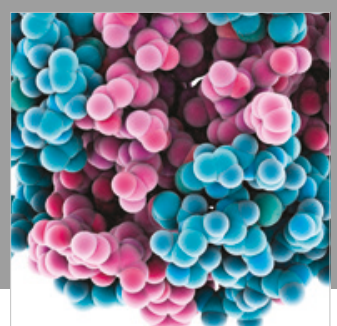

ournal of

Diabetes Research

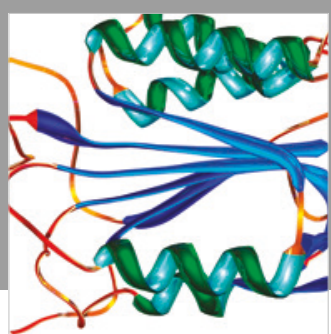

Disease Markers
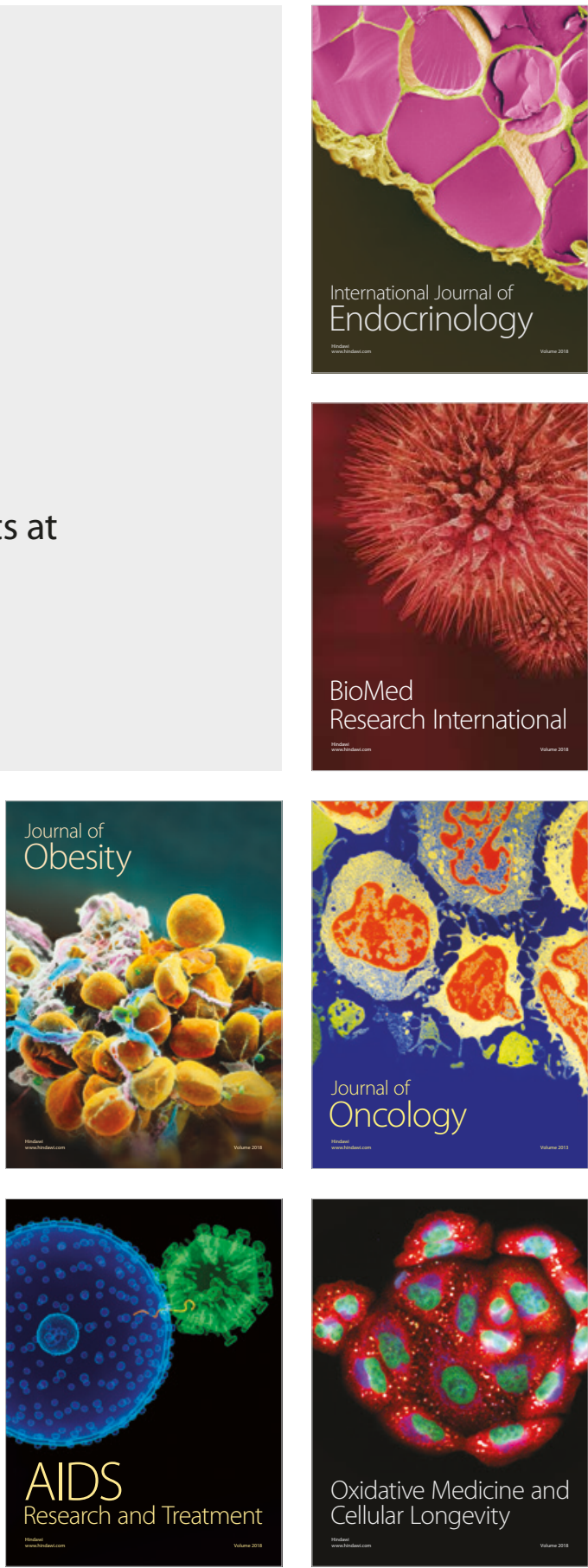